LA-UR-99-3216

Approved for public release:

distribution is unlimited.

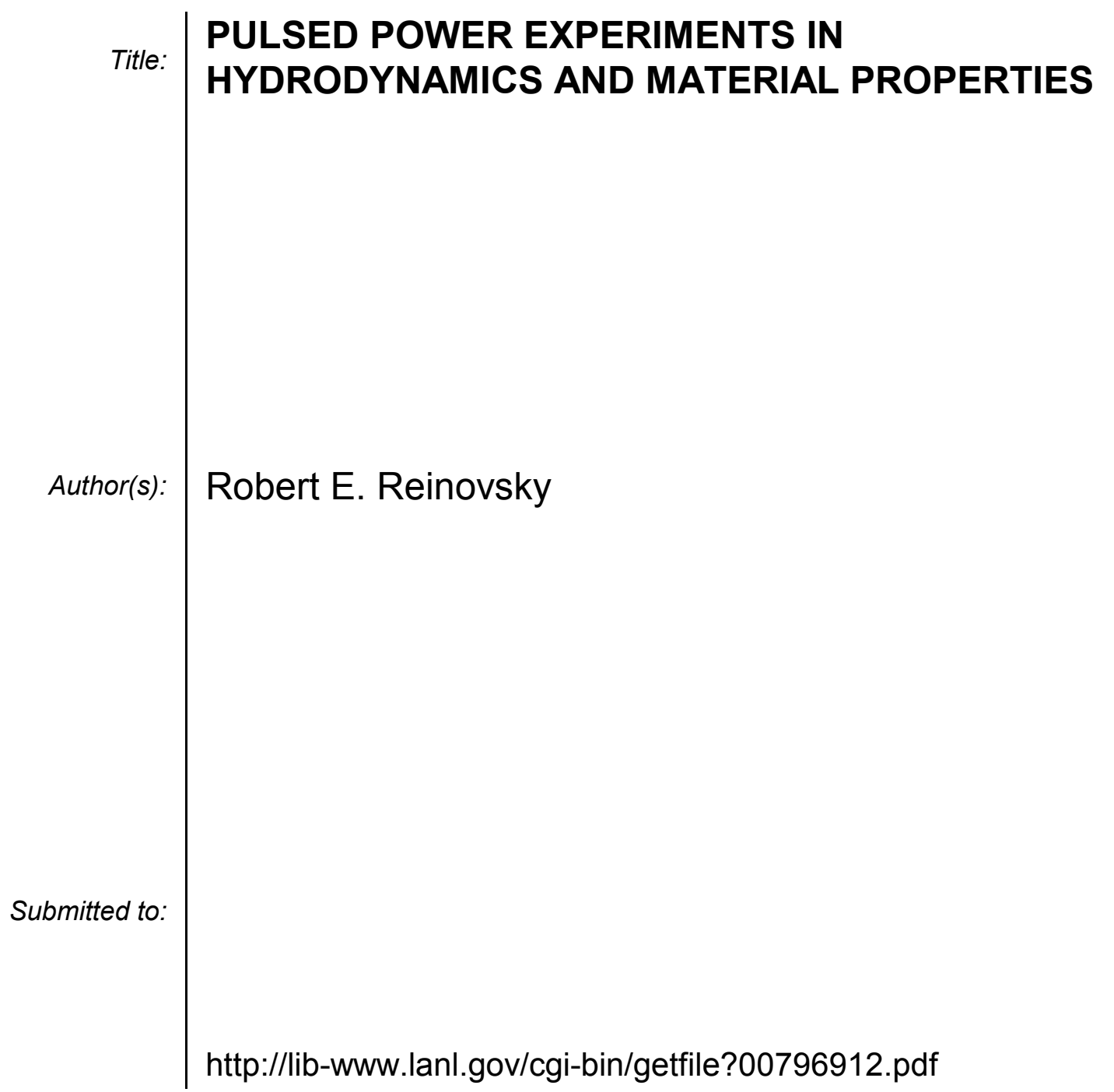

Los Alamos National Laboratory, an affirmative action/equal opportunity employer, is operated by the University of California for the U.S. Department of Energy under contract W-7405-ENG-36. By acceptance of this article, the publisher recognizes that the U.S. Government retains a nonexclusive, royaltyfree license to publish or reproduce the published form of this contribution, or to allow others to do so, for U.S. Government purposes. Los Alamos National Laboratory requests that the publisher identify this article as work performed under the auspices of the U.S. Department of Energy. Los Alamos National Laboratory strongly supports academic freedom and a researcher's right to publish; as an institution, however, the Laboratory does not endorse the viewpoint of a publication or guarantee its technical correctness. 


\title{
PULSED POWER EXPERIMENTS IN HYDRODYNAMICS AND MATERIAL PROPERTIES*
}

\author{
Robert E. Reinovsky \\ Los Alamos National Laboratory \\ PO Box 1663, MS D420 \\ Los Alamos, New Mexico 87545
}

\section{Abstract}

In the last five years, a new application for a high performance pulsed power program has joined the traditional family of radiation source applications in the Stockpile Stewardship. This new application is the production of high energy density environments in materials for the study of material properties and hydrodynamics in complex geometries. The principle tool for producing high energy density environments is the high precision, magnetically imploded, near-solid density liner. The most attractive pulsed power system for driving such experiments is an ultra-high current, low impedance, microsecond time scale source that is economical both to build and operate.

Two families of pulsed power systems can be applied to drive such experiments. The 25-MJ Atlas capacitor bank system currently under construction at Los Alamos is the first system of its scale specifically designed to drive high precision solid liners. Delivering $30 \mathrm{MA}$, Atlas will provide liner velocities $12-15 \mathrm{~km} / \mathrm{sec}$ and kinetic energies of $1-2 \mathrm{MJ} / \mathrm{cm}$ with extensive diagnostics and excellent reproducibility. Explosive flux compressor technology provides access to currents exceeding $100 \mathrm{MA}$ producing liner velocities above $25 \mathrm{~km} / \mathrm{sec}$ and kinetic energies of $5-20 \mathrm{MJ} / \mathrm{cm}$ in single shot operations.

In this paper we will review basic scaling arguments that set the scope of the environments available with pulsed power drive. We will overview the pulsed power technology under development at Los Alamos for high energy density experiments and provide a summary of results from exploration of the physics limiting the performance of near solid metal liners under magnetic drive. We will present few examples of hydrodynamic experiments performed with interim systems.

\section{INTRODUCTION}

The last few years have seen a new application for high performance pulsed power supporting the Stockpile Stewardship program added to the traditional family of radiation source applications. This new application is the use of pulsed power to produce high energy density environments for the study of material properties under extreme conditions and of hydrodynamics in complex geometries. The principle tool for producing the high energy density environments is the high precision, magnetically imploded, near-solid density liner. The most attractive pulsed power system for driving such experiments is an ultra-high current, low impedance, microsecond timescale source that is both economical to build and reliable to operate.

Magnetically imploded liners can produce a variety of high energy density environments. When imploded in free flight to velocities above $10 \mathrm{~km} / \mathrm{sec}$ and kinetic energies of from 1 to $25 \mathrm{MJ} / \mathrm{cm}$ of height, liners are attractive impactors for driving strong shocks in the target. Liner systems can deliver multiple shocks to the target, either by employing multiple liners, or by employing reflected shocks, allowing access to both Hugoniot and off-Hugoniot conditions. When filled with a suitably compressible material, for example a magnetized fusion plasma, liners can deliver almost adiabatic compression to the target, converting its kinetic energy into internal energy and dramatically heating the target. When the compressible material is a vacuum magnetic field, flux compression can result in compressed fields above 1000 Tesla in macroscopic (mm scale) volumes. And when the liner surrounds a (small) nearly incompressible target material, for example a condensed noble gas, a liner can deliver enormous pressure to the target, almost isentropically, without strong shocks.

\section{LINERS}

Magnetically imploded liners offer unique advantages for high energy density materials experiments. Because energy is delivered to the liner from the driving magnetic field at the speed of light, magnetically imploded liners can reach velocities higher than those available from gas guns or planar explosive systems. Higher velocity in the liner-

\footnotetext{
${ }^{*}$ Work supported by US DOE.
} 
impactor means higher pressures and temperatures in the target. Because the parameters of the electrical drive can be continuously adjusted over a wide range, (unlike chemical explosive) the liner acceleration profile and hence final velocity can be continuously and controllably varied to meet experimental requirements. Furthermore, with appropriate design, the acceleration delivered by the magnetic field to the liner is nearly shockless, allowing the condition of the liner to be well characterized upon impact with the target. Compared to high explosive systems, the magnetic driving field is uniform, and completely free from the imprint of high explosive initiation systems. The size scale of magnetically driven liners naturally couples to centimeter scale target volumes $(2 \mathrm{~cm} \mathrm{x} 2 \mathrm{~cm})$ in current systems. Centimeter scale targets allow experiments to be performed in which the target is many times the characteristic grain size of the material. Excellent azimuthal symmetry and axial uniformity have been achieved, allowing high precision experiments to be planned. And the fundamentally cylindrical geometry permits good diagnostic access both down the axis of the cylinder and transverse to it. The cylindrical geometry allows the establishment of a variety of special initial conditions such as the introduction of a pre-ionized plasma into the interior of the cylinder.

For high energy density hydrodynamics experiments, the first requirement is that the liner arrives at the target with most of its mass near normal density and preferable with the inner surface of the liner unmelted. The most elementary model of liner behavior is that of a simple inductive store carrying an initial current adequate to store the required magnetic energy. Current delivered from the storage inductor implodes the liner but magnetic flux is conserved in the storage inductor/liner system. This allows the kinetic energy, velocity and implosion dynamics to be expressed analytically as functions only of the value of the storage inductor and the liner dimensions (initial and final radius, height and thickness). When constrained by average action in the liner $\left(3 \times 10^{16}\right.$ for melt in aluminum) this analytic formulation allows the region in velocity/kinetic energy space accessible with magnetically imploded liners to be described.

For magnetically stored energies of $25 \mathrm{MJ}, 100 \mathrm{MJ}$, and $200 \mathrm{MJ}$ and storage inductors ranging from $2 \mathrm{nH}$ to $20 \mathrm{nH}$, initial currents in the inductor of $100 \mathrm{MA}$ to $316 \mathrm{MA}$ are required. As shown in Fig. 1, under these conditions, velocities around 20,30, and $40 \mathrm{~km} / \mathrm{sec}$ with kinetic energies of 5,15 , and $30 \mathrm{MJ} / \mathrm{cm}$ of height can be achieved, respectively. ${ }^{1}$

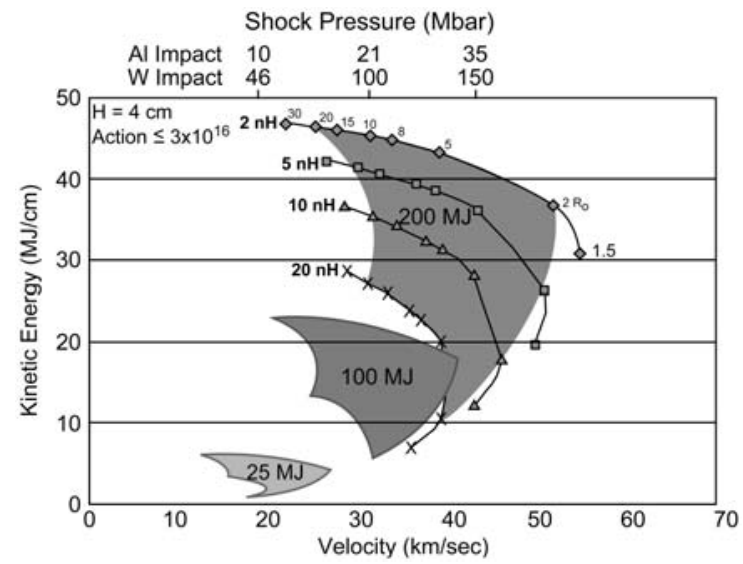

Fig. 1. Parameter space accessible with high precision liners.

Requirements on the condition of the liner vary from experiment to experiment but most applications share the need for a high degree of symmetry and uniformity in the liner after implosion. A variety of analytic and 1D and 2D computational tools have been applied to predict the behavior of magnetically imploded cylindrical liners. As shown in Fig. 2, the behavior is not simple. As in all z-pinches, the outer surface of a magnetically imploded liner is unstable to the growth of small perturbations through the magneto-hydrodynamic Rayleigh-Taylor (RT) instability during acceleration. Therefore, initially, small imperfections can grow to become large-scale distortions making liners unusable for many experiments. On the other hand, material strength in the liner should, from first principles, reduce the growth rate of RT modes - and material strength can render some combinations of wavelength and amplitude analytically stable. Unfortunately, the same large currents that drive the liner also ohmically heat the outer surface resulting in strengthless fluid layer at the unstable field/liner interface. In some cases the liner may be solid through most of its thickness when it arrives at the target. In other cases it is completely melted. The growth of instabilities in both soft aluminum liners and in high strength aluminum alloy liners has been studied analytically, computationally, and experimentally at liner kinetic energies up to $100 \mathrm{KJ} / \mathrm{cm}$ on the Pegasus capacitor bank using driving currents up to $12 \mathrm{MA}$. Experiments have been performed in which $\mathrm{m}=0$ (sausage mode) perturbations of amplitudes 10-100 micron and wavelengths from a fraction to a few $\mathrm{mm}$ have been introduced on the outer surface of the liner. 


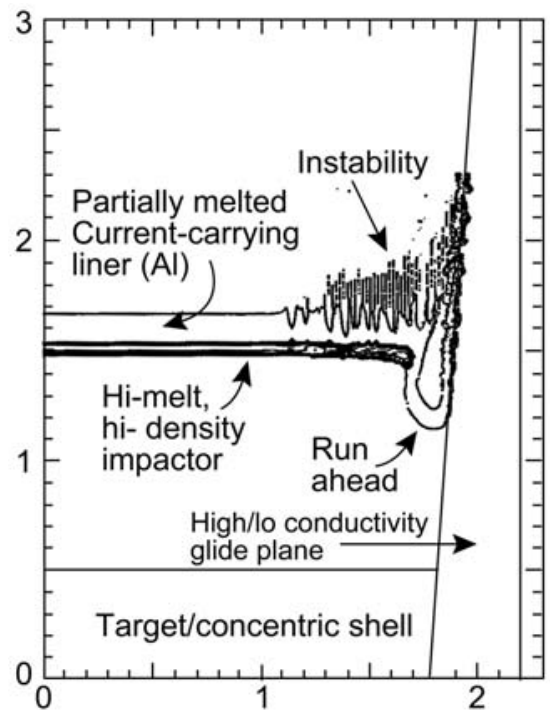

Fig. 2. 2D MHD calculations predict complex liner behavior.

Growth of the perturbations have been monitored radiographically as shown in Fig. 3 and compared with analytical and 2D simulation. ${ }^{2}$ Notably good agreement between simulation and experimental radiographs has been observed as shown in Fig. 4

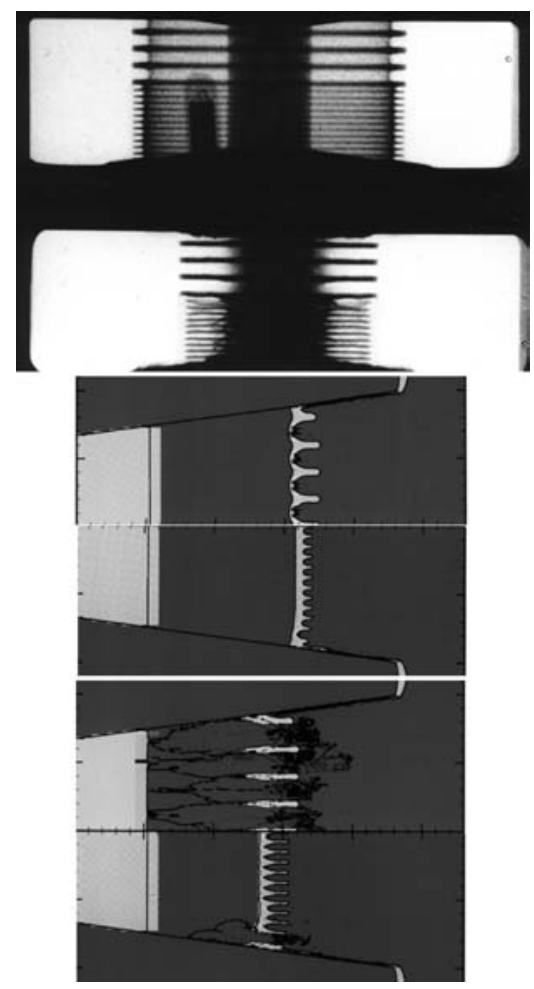

Fig. 3. Radiograph of perturbation growth on outer surface of liner compared with 2D MHD calculations.

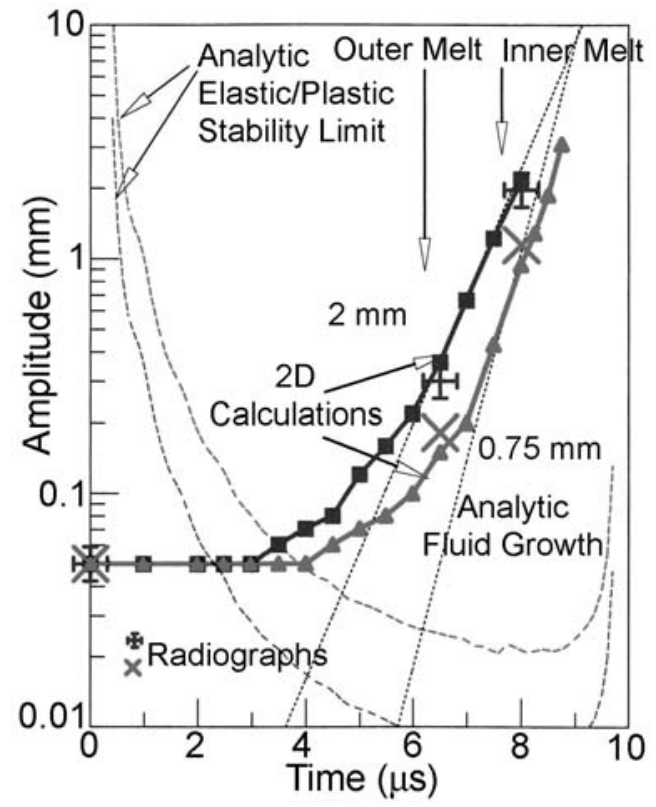

Fig. 4. Experimentally determined perturbation growth corresponds well with 2D predictions. Analytic models describe limiting behavior.

Material strength analytically stabilizes the RT instability and perturbation growth experiments have been conducted with aluminum alloy liners displaying up to an order of magnitude higher (static) yield strength than that of nominal 1100 aluminum. ${ }^{3}$ Unfortunately, higher strength alloys also display larger electrical resistivity and a new phenomena called the resistive explosion instability has emerged to limit performance of higher strength lines. This instability is characterized by non-uniform ohmic heating and nonuniform field penetration in the perturbation. These result in non-uniform melting and growth of the instability at rates faster than that predicted by linear theory.

Figure 5 shows the results from one analytic formulation for perturbation growth in strong materials for accelerations characteristic of Pegasus and Atlas experiments. Experiments comparing effects of surface finish have been performed and compared with the analytic formulations for instability growth. The results of these experiments indicate that the limits imposed by long wavelength (mm scale) "waviness" in the liner will be more severe than the limits imposed by short wavelength $(<100-\mu$ scale) surface finish. Experiments have also been conducted to explore the stabilizing effects of adding concentric cylindrical shells. Reduction of perturbations on the inner surface of the second shell is observed, and 2D calculations generally described the experimental results. Finally, one experiment has been performed to assess the effects of 
applying the drive magnetic field oblique to the perturbations with the result that instability growth was apparently dramatically reduced.

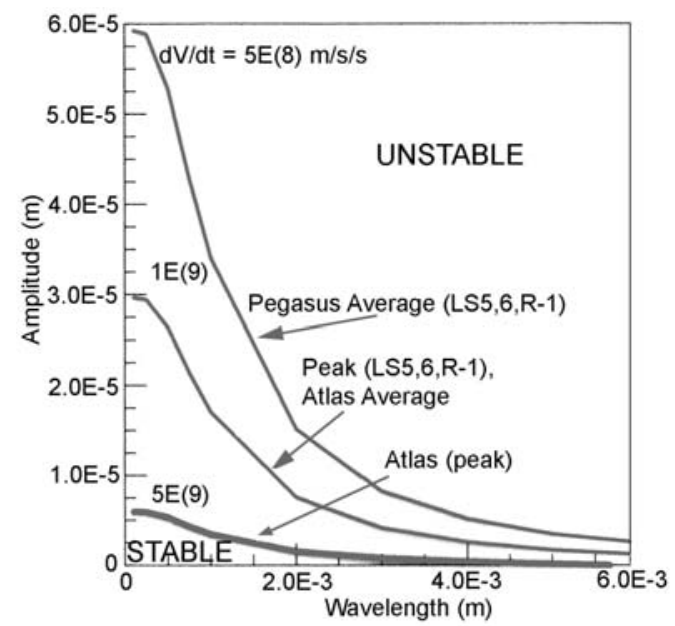

Fig. 5. Wavelength/amplitude space showing stability boundaries for one analytic formulation of perturbation growth.

In general it appears that an adequate suite of experimental tools, analytic and simulation techniques are available to predict liner behavior.

\section{PULSED POWER FOR HYDRODYNAMIC EXPERIMENTS}

The most significant pulsed power performance issues for high energy density hydrodynamics experiments is the delivery of energy at the highest practical currents (lowest impedance) with time-scales matched to the experiments. For liners of radius about $5 \mathrm{~cm}$ and final velocities of $1-2 \mathrm{~cm} / \mu \mathrm{s}$, characteristic implosion times are 5-10 $\mu$ s and average accelerations are $1-2 \times 10^{10} \mathrm{~m} / \mathrm{s} / \mathrm{s}$. High performance, low inductance capacitor banks conveniently meet these drive requirements for currents up to 30-40 MA. For larger currents, fast explosively driven flux compressors have been demonstrated to drive imploding liners with currents exceeding $100 \mathrm{MA}^{4}$ At Los Alamos, experiments are currently being performed on the Pegasus 4.3-MJ fast capacitor bank. Pegasus delivers up to $12 \mathrm{MA}$ to imploding liner experiments with approximately $6-\mu$ s current rise time. Similarly, fast flux compressors in both $\operatorname{disk}^{5}$ and coaxial ${ }^{6}$ configurations are used for driving experiments at higher current. The Atlas ${ }^{7}$ pulsed power system (Fig. 6) is currently under construction and is scheduled for commissioning in early 2001. It will store $24 \mathrm{MJ}$ in 96 Marx generators operating at peak erected voltage of
$240 \mathrm{KV}$. Current is delivered to the load through parallel (vertical) oil insulated tri-plate transmission lines and an oil insulated convolute connects the transmission lines to the disk transmission lines driving the liner.

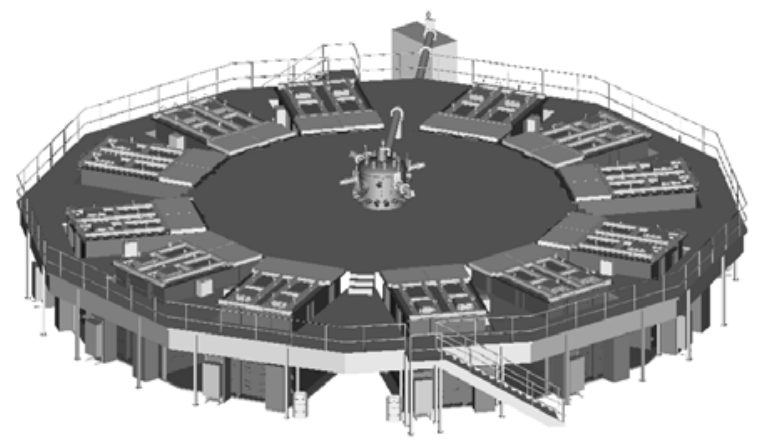

Figure 6. The Atlas pulsed power system.

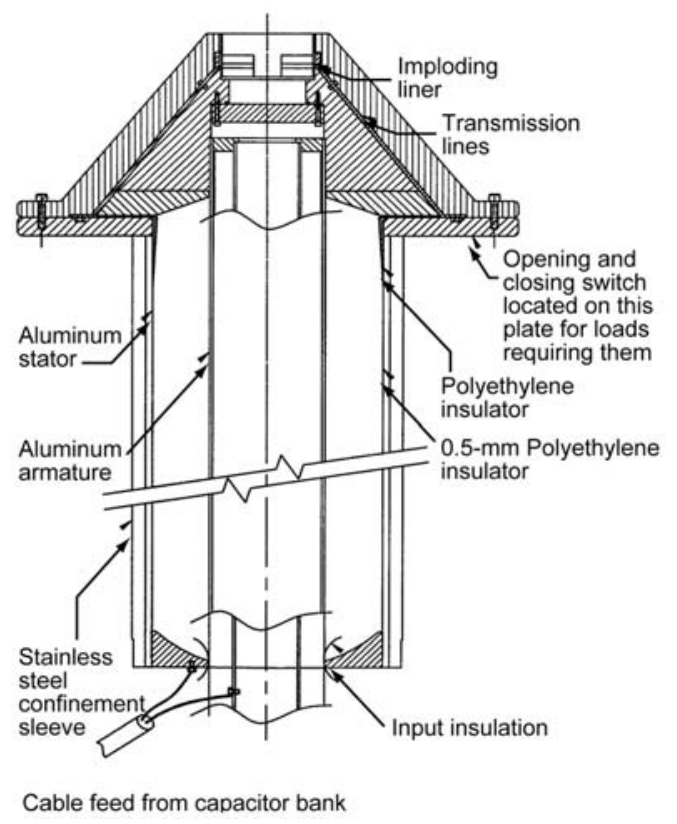

Fig. 7. The Ranchero explosive pulsed power system.

While explosive flux compression systems provide access to parameters currently unattainable in laboratory systems, they also provide economical access to more modest parameters on a limited basis. The Ranchero system is being used to address transmission line, power flow, and liner physics issues at Atlas parameters (30 MA) during the development and construction phases of the Atlas project. The Ranchero system has successfully driven a nominal Atlas liner through a nominal insulator interface and a conceptual solid insulated bi-conic transmission line. The Ranchero generator has also delivered Atlas electrical parameters to a 5-nH load with rise time somewhat faster than Atlas. Ranchero will permit almost two 
years of power flow system validation and liner performance characterization prior to the commissioning of Atlas. This dual approach will permit Atlas to perform hydrodynamic experiments shortly after its commissioning.

\section{HYDRODYNAMIC EXPERIMENTS}

Experiments planned for Atlas fall into three categories: a) those exploring the properties of (near) normal density materials at extreme pressure and temperature; b) those exploring the hydrodynamic behavior of imploding systems; and c) those investigating the properties and behavior of dense plasmas. In the first category, pulsed power driven liner experiments can explore the equation of state of materials under single shock (Hugoniot) and multishock (off Hugoniot) conditions. Liners reach material strains and strain rates exceeding those available from other techniques. Issues in implosion hydrodynamics include instability growth in full strength and strengthless materials, the behavior of material at interfaces (friction), and hydrodynamic flows in complex geometries. Plasmas in which the ion and electron physics are strongly coupled are difficult to produce and little experimental data is available. With pulsed power experiments plasmas characterized by $\Gamma>5$ and $\Theta \sim 1$ can be produced for study of EOS and transport properties, instability growth at interfaces and hydrodynamics in simple and complex geometries.

\section{A. Material Properties}

In the area of material properties experiments, pulsed power driven liners offer unique access to simultaneous conditions of high strains and strain rate in macroscopic samples. Because of cylindrical convergence, the inner surface of an imploding Atlas liner can reach strains exceeding $200 \%$ at strain rates of $10^{4}$ to $10^{6}$ per second - a regime unreachable by any other technique. By proper choice of liner parameters (usually a high conductivity aluminum armature surrounding a thinner cylinder of the material of interest), the test sample can be isolated from the ohmic heating of the driving current and the acceleration applied in such a way as to insure that the sample material is not shocked. Thus the temperature of the sample is determined strictly by distortional heating. For materials tested to date on Pegasus, $\mathrm{Al}, \mathrm{Ta}, \mathrm{Cu}$, and $\mathrm{SS}$, temperature rises of a few hundred Kelvin are predicted. The temperature of the inner surface is measured during implosion using a multi-band infra-red pyrometer with uncertainty of about $\pm 50 \mathrm{~K}$. Temperature rise as a function of time (radius or average strain) compares favorably, but not precisely with that predicted by various material strength models.

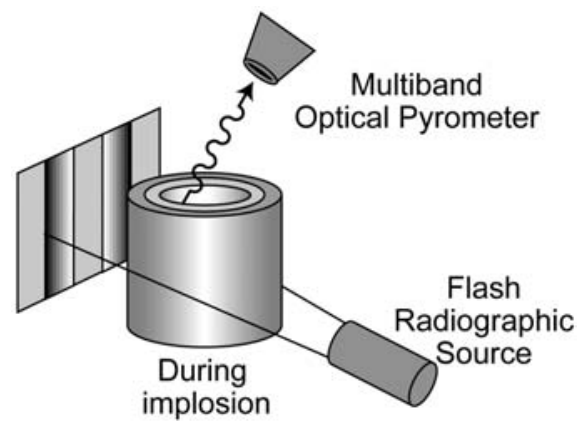

Fig. 8. Configuration of an experiment performed on

Pegasus to evaluate strength effects through distortional heating of the inner surface of a liner.

\section{B. Implosion Hydrodynamics}

The imploding liner is also an excellent driver for experiments exploring the differential motion of material at interfaces and the growth of hydrodynamic instabilities. In one family of experiments already conducted on Pegasus, an imploding liner impacts an azimuthally segmented (pie sectioned) target in which adjacent sections are alternately high-density tantalum and low-density aluminum. As shown in Fig. 9, fractional $\mathrm{mm}$ diameter lead marker wires are embedded in the aluminum and the target is radiographed axially as the shock converges and then reflects from the axis. Since the shock runs substantially faster in the aluminum, there is a relative motion at the interface of a fraction to a few $\mathrm{mm} / \mu \mathrm{s}$. Development of the boundary layer motion is diagnosed by curvature and distortion of the wires. The experiment is performed as a function of shock strength (relative interfacial velocity), materials, surface condition, and bonding.

In another experiment, the liner impacts a hollow target filled with gas or low-density foam. Small perturbations are imposed on the inner surface of the metal target. The magnitude and time history of the pressure pulse in the material is determined by liner impact conditions and by the introduction of a shock "receiver" material (acrylic) between liner and target. Again by radiographing down the axis of the target (Fig. 10), unstable growth of the perturbations is measured and compared with predictions. Furthermore, cylindrical layers of alternating normal density and 
reduced $\left(1 \mathrm{gr} / \mathrm{cm}^{3}\right)$ aluminum are observed as the target material exceeds its "spall" strength.

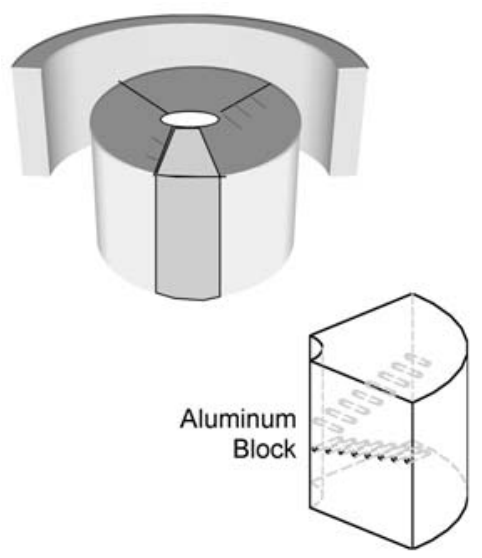

Fig. 9. Configuration of an experiment performed on Pegasus to explore the development of boundary layer flow at an interface with shock driven differential motion.

\section{Properties of Dense Plasmas}

The cylindrical geometry of pulsed power driven liner experiments also makes it possible to introduce materials within the liner in a variety of interesting initial conditions. For example, a moderate density iron plasma created by the electrical explosion of an array of aluminum wires can be compressed between the driving liner and a center "anvil" to become a strongly correlated plasma.

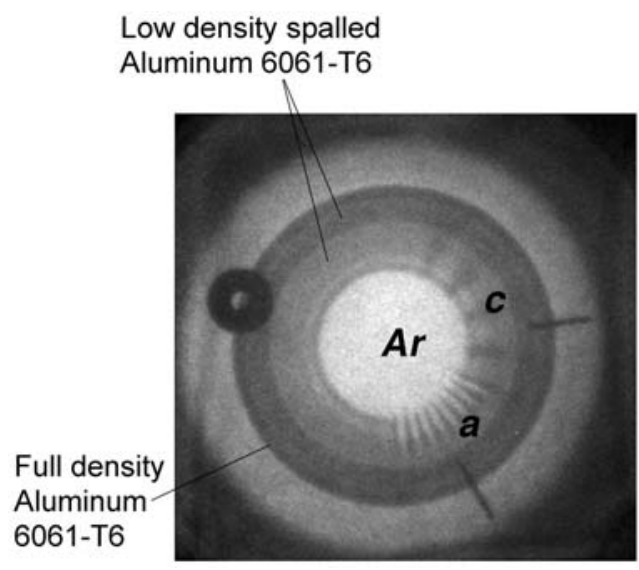

Fig. 10. Results of an experiment performed on Pegasus to explore instability growth in a shock accelerated interface.

In a different vein, the liner can be filled with a magnetized fusion plasma initially formed at number density of $10^{18} / \mathrm{cm}^{3}$ and $100-200 \mathrm{eV}$ and compressed by the liner at velocities of $1-2 \mathrm{~cm} / \mu \mathrm{s}$ to reach fusion ignition temperatures.

\section{SUMMARY}

The development of economical, highly reliable, lowimpedance capacitor banks coupled to high precision near solid density liners imploding at $10-20 \mathrm{~km} / \mathrm{sec}$ have made possible the development of a wide variety of hydrodynamic experiments. The uniformity, controllability, and high liner velocities enable experiments not otherwise possible and represent a complement to lasers and nanosecond pulsed power used for radiation driven experiments. The addition of simple, though single-use, explosive magnetic flux compressors to deliver another order of magnitude more energy allowing experiments to be extended into regimes of extreme energy density on multi-centimeter target scales.

\section{REFERENCES}

${ }^{1}$ R. E. Reinovsky, C. A. Ekdahl, "Development of Imploding Liners with Kinetic Energies >100 MJ and their Applications," in

Proceedings of $7^{\text {th }}$ International Conference on Megagauss Magnetic Field Generation and Related Topics, 1996, p. 660.

${ }^{2}$ R. E. Reinovsky, et al., "Stability of Magnetically Imploded Liners for High Energy Density Experiments," in Proceedings of $8^{\text {th }}$

International Conference on Megagauss Magnetic Field Generation and Related Topics, 1998, to be published.

${ }^{3}$ A. M. Buyko, et al., "Experimental Investigation of Characteristics of the Cylindrical Liner With Sinusoidal Perturbations Imploding Under the Effect of Magnetic Field," in Proceedings of $8^{\text {th }}$ International Conference on Megagauss Magnetic Field Generation and Related Topics, 1998, to be published.

${ }^{4}$ R. E. Reinovsky, et al., "HEL-1: A DEMG Based Demonstration of Solid Liner Implosions at 100 MA," in Proceedings of the $11^{\text {th }}$ International Pulsed Power Conference, 1997, p. 378.

${ }^{5}$ R. E. Reinovsky, et al., "Caballero: A High Current Flux Compressor System for 100-MJ Solid Liner Experiments," in Proceedings of the $11^{\text {th }}$ International Pulsed Power Conference, 1997, p. 1448.

${ }^{6}$ J. H. Goforth, et al., "The Ranchero Explosive Pulsed Power System," in Proceedings of the $11^{\text {th }}$ International Pulsed Power Conference, 1997, p. 1573.

${ }^{7}$ R. B. Miller, et al., "The Atlas Pulsed Power Project," in Proceedings of the $12^{\text {th }}$ International Pulsed Power Conference, 1999 (this conference) to be published. 